\title{
CROSS-CULTURAL ADAPTATION AND PSYCHOMETRIC PROPERTIES OF A MUTUAL UNDERSTANDING SCALE (MUS) - INDONESIA VERSION
}

\author{
Yohanes Tebai', Diah Ayu Maharani', Ilya Revianti Sudjono Sunarwinadi' ${ }^{2}$ Anton Rahardjo' \\ 'Department of Preventive and Public Health Dentistry, Faculty of Dentistry, Universitas Indonesia, Jakarta, Indonesia \\ ${ }^{2}$ Department of Communication, Faculty of Social and Political Sciences, Universitas Indonesia, Depok, Indonesia
}

\begin{abstract}
INTRODUCTION: Transactional communication between a doctor/dentist and a patient is important. Doctors and patients should equate clinical realities or explanatory models in health consultations and communicate in accordance with mutual understanding.

Овјестіves: The aims of this study were to cross-culturally adapt the Mutual Understanding Scale (MUS) to Indonesian language and to test its reliability and validity.

MATERIAL AND METHODS: MUS is a questionnaire for measuring mutual understanding; however, the oral health services version and Indonesian version of the scale is not yet available. In this study, we assessed the validity and reliability of an Indonesian version of MUS for assessment of mutual understanding between dentist and patients in oral health service at Jakarta. A sample of patients was recruited from among 100 Papuan with oral health complaints. After translating the instrument, we conducted psychometric testing.

RESULTS: The reliability coefficients (Cronbach's $\alpha$ ) for Indonesian version of MUS dentist and MUS patient questionnaires were 0.928 and 0.925 , respectively. The test-retest reliability test of MUS Indonesian version was rated as excellent interclass correlation coefficient for MUS dentist and MUS patient as 0.845 and 0.825 , respectively. With respect to validity, the scores of MUS Indonesian version showed significant correlation coefficient $r=0.70-0.80$, with global ratings on a five-point Likert scale. The perception with global rating dichotomies questions was defined as significant in Mann-Whitney test, with $p<0.05$.

ConcLusions: The questionnaire showed excellent discriminant validity for measurement of mutual understanding of dentists and patients in dentist-patient communications. This study provides strong evidence of the reliability and validity of the Indonesian MUS for assessment of mutual understanding during dentist-patient communication in oral health services.
\end{abstract}

KEY WORDS: mutual understanding, communication, reliability, validity.

J Stoma 2018; 71, 4: 315-321

DOI: https://doi.org/10.5114/jos.2018.83404

\section{INTRODUCTION}

Transactional communication between doctor/dentist and patients is essential to achieve a mutual understanding [1-3]. Health and oral health consultations and ser- vices require effective communication to attain a mutual understanding between the health professional and the health service patient [4]. However, this element of consultation is often neglected [3]. Transactional communication between a physician and a patient is neces-

\section{JOURNAL OF STOMATOLOGY CZASOPISMO STOMATOLOGICZNE}

AdDRESS FOR CORRESPONDENCE: Anton Rahardjo, Department of Preventive and Public Health Dentistry, Faculty of Dentistry, Universitas Indonesia, Jakarta 10430, Indonesia, e-mail: anton_r@ui.ac.id 
sary to collaborate on the cause of the disease, choice of care, and post-care instructions, both according to the physician's judgment and feelings and expectations of the patient $[5,6]$. According to Kleinman theory, both patients and doctors have their own clinical reality and communication regarding the disease and health care [7].

Many studies have shown that cultural differences are a barrier to mutual understanding and affect the communication between a dentist and a patient $[1,8,9]$. Indonesia is a multi-ethnic and multicultural country. Different cultural background of dentists and patients may contribute to lack of effective communication between a dentist and a patient $[1,4]$. Frequently, the dentist communicates with his patients who have different views on oral health and dissimilar culture in providing oral health services [10]. Mutual Understanding Scale (MUS) is a questionnaire to assess the perceived mutual understanding in intercultural interactions [3]. This questionnaire is particularly relevant in the multicultural context of Indonesia. However, there is no suitable version of this questionnaire that appropriately reflects the sociocultural context and health services in Indonesia. Recent study shows that MUS is only ready for medical service, not for oral health services. In this study, we adapted the MUS questionnaire for assessment of perceived mutual understanding between dentists and patients during oral health service (OHS) consultations in Indonesia. Psychometric testing of the Indonesian version of MUS questionnaire was conducted in a study on Papuan students in Jakarta who received oral health consultations from non-Papuan indigenous dentists.

\section{OBJECTIVE}

The aims of this study were to cross-culturally adapt the MUS to Indonesian language and to test its reliability and validity.

\section{MATERIAL AND METHODS}

This was a cross-sectional study. The MUS questionnaire consists of two parts: MUS questionnaire for patients and MUS for doctors/dentists; both parts contained the same questions. The first part of the two MUS questionnaires captures the sociodemographic characteristics of the respondents. The second part of both these questionnaires consists of 5 domains: subjective domain, causes of illness, analysis and diagnosis, objective domain, and treatment plans. Items in the subjective domains, causes of disease, and analysis of diagnosis are rated on a 3 -point Likert scale $(-1=$ no understanding or no mutual understanding; $0=$ hesitating; $+1=$ understanding and having mutual understanding). Items in the objective domain and treatment plan are scored on a binary scale $(1=$ yes and $2=$ no). The average value of mutual understanding is classified into two catego- ries, i.e., $\leq 0=$ no mutually perceived understanding; $\geq 0.1=$ presence of mutual understanding [3].

The English version of the MUS questionnaire was originally created and introduced by Harmsen et al. in 2004 [3]. The translation of the questionnaire to Indonesian language and its back-translation into English was performed by lecturers who obtained their medical degree abroad. The translated MUS was assessed and revised by an expert panel to achieve conformity with respect to the concepts and content between the original version and the Indonesian version. The panel consists of a dentist and a community dental researcher who were familiar with the dentist-patient communication. The consensus version of the translated questionnaire was tested in 10 Papuan students in Jakarta and a non-Papuan indigenous dentist to determine the sensitivity to Indonesian culture and the selection of appropriate words. For transcultural adaptation, face-to-face interviews were conducted with Papuan patients who came for dental treatment or oral hygiene consultation at dental clinics in Jakarta. The consensus version was translated back into English. The back-translation of the Indonesian version into English was conducted by three doctoral students at the Faculty of Dentistry, the Universitas Indonesia who were not familiar with the words in the original MUS. Finally, the MUS was confirmed by an expert panel. The back-translated English version was sent to the author of the original MUS who confirmed that there was no change in context and composition of the sentences.

Sample size estimation suggested that a cell size of 100 subjects completing the study will be sufficient to detect statistically significant differences $(p<0.05)$ with a power of $95 \%$, assuming a significant correlation of 0.4. Inclusion criteria involved present or past history of toothache or any oral diseases.

Four dentists were involved in this study in Jakarta. One dentist attends 9 or more patients, and one patient spends 7-15 minutes in communication and oral health consultation.

The test-retest reliability of the questionnaire was assessed in 10 patients ( $10 \%$ of the total respondents) who underwent repeated interview with questionnaires within one week of the first measurement. Reliability was tested using Cronbach's $\alpha$ and interclass correlation coefficient (ICC). The validity of concepts was assessed by testing the association between MUS scores and global rankings using Spearman's correlation coefficient. Discriminant validity was tested by comparing MUS score with global ratings using Mann-Whitney test.

The global ranking questionnaire includes global ranking questions. The global question is a question that represents all questions in MUS. The validity was assessed using a global question for both MUS dentist and MUS patients' questionnaires. For the MUS dentist, the global question was: "Overall, do you understand your conversation with your patients?”. The global ques- 
tion for MUS patients was: "Overall, do you understand your conversation with your dentist?". The response options were: 1 - strongly understand; 2 - do not understand; 3 - doubtful; 4 - understand; 5 - strongly understand. The response options correlated with all domains in the MUS questionnaire. The second global question for dentists was: "Are you able to convey your question to the patient without difficulty?". The response options: 1 - no; 2 - yes, compared to all MUS domain values.

The principal investigator agreed to provide direct access to source data/documents for study-related monitoring, audits, IRB/IEC review, or regulatory inspection, if required. This research was approved by the Dental Research Ethical Committee at the Faculty of Dentistry, University of Indonesia (No. 20/Ethical Approval/ FKGUI/IV/2017 Protocol Number: 070210317). Written informed consent was obtained from all subjects prior to their enrolment. Questionnaires were administered to subjects, with each question having five options for an answer. The time to complete the questionnaire was about 20 minutes. Ten out of the 100 subjects (10\%) were asked to complete the questionnaire twice for assessment of test-retest reliability. The total duration of the first data collection and second data collection was 14 days. Personal information such as age, sex, education level, occupation, ethnicity, marital status, and other demographic data were collected.
Assessment of mutual understanding between the dentist and the patient was completed after dental consultation and communication in OHS with MUS Indonesia version. Inform consent was only obtained at the beginning of the measurement. All subjects were explained about the study prior to the questionnaire survey. Measurements were taken after 3-8 days after OHS. The data obtained were subjected to statistical analysis.

\section{RESULTS}

The content of the translated and original version of the MUS were similar. Only minor differences were identified between the back-translated and the original version. No specific issues were identified during the translation and back-translation processes. Therefore, no modification was done on the original version of the MUS. Completed usable questionnaires were received from all 100 respondents (100\% response rate). The average age of the respondents was 22 years (range, 18-24).

A prominent feature of the study sample was that the majority of the respondents were male $(84.8 \%)$ and only $4 \%$ respondents had spent money below the Papuan capital rate. There were no significant differences between the two groups with respect to any of characteristics $(p>0.05)$ (Table 1). Many Papuan women were better

TABLE 1. Characteristics of respondents disaggregated by presence or absence of mutual understanding, $N=100$

\begin{tabular}{|c|c|c|c|}
\hline Factor & No MU & MU & $p$ value \\
\hline Age (years) & $19(19-20)$ & $20(17-23)$ & $0.62^{* *}$ \\
\hline \multicolumn{3}{|l|}{ Education level* $^{*}$} & \multirow[t]{3}{*}{$0.88^{*}$} \\
\hline Primary and secondary & $15(45.5)$ & $33(47.30)$ & \\
\hline Tertiary & $18(54.5)$ & $34(50.71)$ & \\
\hline \multicolumn{3}{|l|}{ Sex* } & \multirow[t]{3}{*}{$0.17^{*}$} \\
\hline Male & $28(84.8)$ & $47(70.10)$ & \\
\hline Female & $5(15.2)$ & $20(29.91)$ & \\
\hline \multicolumn{3}{|l|}{ Ethnicity and language by region* } & \multirow[t]{3}{*}{$0.20^{*}$} \\
\hline Rural & $20(60.6)$ & $30(44.80)$ & \\
\hline Border & $13(39.4)$ & $37(55.22)$ & \\
\hline \multicolumn{3}{|l|}{ Spending per month (IDR)*** } & \multirow[t]{3}{*}{$0.43^{*}$} \\
\hline$<480$ & $4(12.1)$ & $4(6)$ & \\
\hline$>480$ & $29(87.9)$ & $63(94)$ & \\
\hline \multicolumn{3}{|l|}{ History of dental consultation* } & \multirow[t]{3}{*}{$0.88^{*}$} \\
\hline Never & $16(48.50)$ & $35(52.21)$ & \\
\hline Always & $17(51.05)$ & $32(47.80)$ & \\
\hline
\end{tabular}


TABLE 2. Mutual understanding of dentist and patient, $N=100$

\begin{tabular}{|l|c|c|c|c|c|c|}
\hline \multirow{2}{*}{ Domain } & \multicolumn{2}{c}{ MUS patient } & \multicolumn{2}{c}{ MUS dentist } & \multicolumn{2}{c|}{ Mutual understanding } \\
& No MU & MU & No MU & MU & value* \\
\hline Subjective; MUS 1,2 & $35(35)$ & $65(65)$ & $33(33)$ & $67(67)$ & $65(97)$ & 0.000 \\
\hline Causative; MUS 3,4,5 & $31(31)$ & $69(69)$ & $33(33)$ & $67(67)$ & $59(96.70)$ & 0.000 \\
\hline Analysis/diagnosis; MUS 6,7 & $39(39)$ & $61(61)$ & $39(39)$ & $61(61)$ & $67(97.10)$ & 0.000 \\
\hline Objective; MUS 8, 9, 10, 11 & $44(44)$ & $56(56)$ & $44(44)$ & $56(56)$ & $56(100)$ & 0.000 \\
\hline MUS 8 & $29(29)$ & $71(71)$ & $34(34)$ & $66(66)$ & $66(93)$ & 0.000 \\
\hline MUS 9 & $26(26)$ & $74(74)$ & $29(29)$ & $71(71)$ & $71(95.90)$ & 0.000 \\
\hline MUS 10 & $36(36)$ & $64(64)$ & $41(41)$ & $59(59)$ & $59(92.20)$ & 0.000 \\
\hline MUS 11 & $35(35)$ & $65(65)$ & $36(36)$ & $64(64)$ & $62(95.42)$ & 0.000 \\
\hline Treatment plan; MUS 12, 13, 14 & $88(88)$ & $12(12)$ & $88(88)$ & $12(12)$ & $12(100)$ & 0.000 \\
\hline MUS 12 & $34(34)$ & $66(66)$ & $14(41)$ & $59(59)$ & $59(89.43)$ & 0.000 \\
\hline MUS 13 & $11(11)$ & $89(89)$ & $14(14)$ & $86(86)$ & $84(94.42)$ & 0.000 \\
\hline MUS 14 & $78(78)$ & $22(22)$ & $78(78)$ & $22(22)$ & $17(77.30)$ & 0.000 \\
\hline
\end{tabular}

Data presented as $N(\%),{ }^{*} \chi^{2}$ test, $p<0.05=$ significant

TABLE 3. Reliability assessment of dentist MUS questionnaire, $N=100$

\begin{tabular}{|c|c|c|c|c|c|}
\hline \multirow{2}{*}{ Domain dentist MUS } & \multicolumn{5}{|c|}{ Reliability } \\
\hline & Mean & SD & a if deleted* & CICTT** $^{* *}$ & Cronbach's a \\
\hline Subjective (S) 1, 2 & 0.375 & 0.891 & 0.892 & 0.931 & 0.928 \\
\hline Causative (C) 3, 4, 5 & 0.443 & 0.738 & 0.879 & 0.966 & \\
\hline Analysis/diagnosis (A) 6, 7 & 0.240 & 0.908 & 0.894 & 0.933 & \\
\hline Objective $(0) 8,9,10,11$ & 1.650 & 0.433 & 0.908 & 0.959 & \\
\hline Treatment plan (P) 12, 13, 14 & 1.556 & 0.250 & 0.957 & 0.672 & \\
\hline
\end{tabular}

${ }^{*}$ Corrected item-total correlation

understood. Comparison of the proportion who understand (20 respondents, 29.9\%) and do not understand (5 respondents, $15.2 \%$ ) in women was more than in men, with not understanding as $28(84.8 \%)$. Out of the 100 respondents (Papuan students), 60 were residing in Jakarta since $\geq 12$ months, while 40 had newly arrived in Jakarta.

Determining the value of MUS from these two domains evaluated the suitability of the answers between the values of the doctor's understanding and the value of the patient's understanding with the $\chi^{2}$ test.

As shown in Table 2, there was a significant difference with respect to the value of mutual understanding between the dentist and the patient. Most dentists declared to understand the communication by Papuan patients, and most Papuan patients stated to understand the communication by dentists during health service consultations. The total proportion of mutual understanding was more common among dentists and Papuan patients.

Responses to MUS 1-7 were recorded on a 3-point Likert scale, while responses to MUS 8-14 were recorded on a 2-point Likert scale. Internal consistency of the MUS questionnaire was assessed by calculating Cronbach's $\alpha$.

As shown in Table 3, the reliability of the dentist MUS questionnaire was rated as very good (Cronbach's $\alpha=$ 0.928). The patient MUS questionnaire also had a high reliability. Both questionnaires exhibited excellent testretest reliability (ICC: 0.845 and 0.825 , respectively). Thus, the dentist MUS questionnaire Indonesian version was found to have a high reliability.

As shown in Table 4, the MUS patient questionnaire showed excellent reliability (Cronbach's $\alpha=0.925$ ).

The face validity of MUS Indonesian version questionnaire was good despite several adjustments of the columns and numbering of MUS questions to facilitate its comprehension by the respondents directly or through a research assistant.

As shown in Table 5, there was a significant correlation $(p<0.05)$ between MUS dentist questionnaire and MUS patients' questionnaire with respect to comprehension of Likert global rank questions (correlation coefficient, $r=0.70-0.80)$. In addition, the dichotomous global rank question had significant differences between 
TABLE 4. Reliability analysis of MUS patient questionnaire, $N=100$

\begin{tabular}{|l|c|c|c|c|}
\hline \multirow{2}{*}{ Domain MUS of patient } & \multicolumn{3}{|c|}{ Reliabilities } \\
\cline { 2 - 5 } & Mean & SD & a if deleted* & CICTT** \\
\hline Subjective (S) 1,2 & 0.37 & 0.89 & 0.89 & 0.931 \\
\hline Causative (C) $3,4,5$ & 0.44 & 0.73 & 0.87 & 0.96 \\
\hline Analysis/diagnosis (A) 6,7 & 0.24 & 0.90 & 0.89 & 0.93 \\
\hline Objective (0) 8, 9, 10,11 & 1.60 & 0.43 & 0.90 & 0.99 \\
\hline Treatment plan (P) 12,13,14 & 1.55 & 0.25 & 0.95 & 0.62 \\
\hline
\end{tabular}

*Cronbach's a if item deleted

**Corrected item-total correlation

TABLE 5. Convergent/construct validity of MUS Indonesian version, $N=100$

\begin{tabular}{|c|c|c|c|c|c|c|c|}
\hline & \multirow{3}{*}{ Domain } & \multirow{3}{*}{ MUS } & \multicolumn{2}{|c|}{ Likert global rank question } & \multicolumn{3}{|c|}{ Dichotomous global rank question } \\
\hline & & & \multirow{2}{*}{$r$} & \multirow{2}{*}{ p value* } & \multicolumn{2}{|c|}{ Median (range) } & \multirow{2}{*}{$p$ value $^{* *}$} \\
\hline & & & & & Able & Disable & \\
\hline \multicolumn{8}{|c|}{ Patient's MUS } \\
\hline \multicolumn{2}{|c|}{ Total score of patients MUS } & $1-14$ & 0.70 & 0.00 & $0.142(0.14-0.50)$ & $0.142(0.79-1.50)$ & 0.00 \\
\hline$S$ & Subjective & 1,2 & 0.78 & 0.00 & $-1(-1-0.00)$ & $1(-1-1)$ & 0.00 \\
\hline C & Causative & $3,4,5$ & 0.75 & 0.00 & $-0.66(-0.67-(-0.33)$ & $1(0.00-1)$ & 0.00 \\
\hline A & Analysis/diagnosis & 6,7 & 0.68 & 0.00 & $1(0.00-1)$ & $1(-1-1)$ & 0.00 \\
\hline 0 & Objective & $8,9,10,11$ & 0.78 & 0.00 & $1(1-1.25)$ & $2(1.50-2)$ & 0.00 \\
\hline P & Treatment plan & $12,13,14$ & 0.78 & 0.00 & $1.333(1-1.33)$ & $1.66(1-2)$ & 0.00 \\
\hline \multicolumn{8}{|c|}{ Dentist's MUS } \\
\hline \multicolumn{2}{|c|}{ Total score of dentist's MUS } & $1-14$ & 0.822 & 0.00 & $0.142(0.14-1.29)$ & $1.428(0.79-1.50)$ & 0.00 \\
\hline$S$ & Subjective & 1,2 & 0.87 & 0.00 & $-1(-1-1)$ & $1(0.00-1)$ & 0.00 \\
\hline C & Causative & $3,4,5$ & 0.89 & 0.00 & $-0.666(-0.67-1)$ & $1(0.00-1)$ & 0.00 \\
\hline A & Analysis/diagnosis & 6,7 & 0.82 & 0.00 & $-1(-1-1)$ & $1(-1-1)$ & 0.00 \\
\hline 0 & Objective & $8,9,10,11$ & 0.87 & 0.00 & $1(1-2)$ & $2(1.50-2)$ & 0.00 \\
\hline$P$ & Treatment plan & $12,13,14$ & 0.72 & 0.00 & $1.33(1-1.67)$ & $1.666(1.33-2)$ & 0.00 \\
\hline
\end{tabular}

understanding and non-understanding of $p<0.05$, with the MUS of patient and MUS of dentists' measurements in determining the patient's and dentists' understanding.

The dichotomous global rank questions in the MUS patient and dentist questionnaires showed significant values $(p=0.000)$ with the Mann-Whitney test. This demonstrates that the Indonesian version of the MUS patient and dentist questionnaire in general and the dichotomous global rank questions in particular, can differentiate groups of Papuan students that can understand and those who do not understand their interaction with the dentist during consultation. The discriminant validity of the MUS patient and MUS dentist questionnaires, i.e., the extent to which this questionnaire can assess mutual understanding between the doctor and the patient by differentiating those who understand from those who do not understand, was statistically significant ( $p=0.00$, Mann-Whitney test).
The results of assessment of discriminant validity of the MUS questionnaires of patients and dentists are shown in Table 6.

The results show that there was a significant difference between respondents who exhibited mutual understanding and those who exhibited no mutual understanding $(p<0.05)$. This indicates that by distinguishing between the non-MU and MU groups, the MUS dentist and patient questionnaires can measure mutual understanding of dentist-patient communication. The MUS of patient and dentist Indonesian version were found to be valid.

\section{DISCUSSION}

According to Kleinmen, a consultation and health communication seek concordance of patient and physi- 
TABLE 6. Results of discriminant validity of MUS, $N=100$

\begin{tabular}{|l|l|c|c|c|c|}
\hline \multicolumn{7}{c}{ Mean (SD) } & Median & Mean (SD) & Median & p value* \\
\hline \multicolumn{7}{|l|}{ Domain } \\
\hline MUS patient
\end{tabular}

cian explanation models. This harmony is essential owing to the differences between the perspectives of doctors and patients, both with respect to the general world-view and views pertaining to health-related issues $[3,11]$. This dissonance is liable to lead to a lack of mutual understanding between the doctor and patient during healthcare consultations [3]. MUS questionnaires can measure the level of patients' understanding, doctors' understanding, and the mutual understanding between dentists and patients, with respect to oral health care communication. Language and cultural differences can act as barriers that impede mutual understanding [4]. The MUS questionnaire is a useful tool for assessment of mutual understanding between doctor and patients in multicultural contexts.

Higher education level facilitates better communication and understanding. The group, which exhibited mutual understanding, had a greater proportion of respondents with higher education level, those who were accustomed to interacting with people of different ethnic origin, and those who were conversant with the use of Indonesian language.

The proportion of women respondents who exhibited understanding was more than that of those who did not exhibit understanding. Among male respondents, the proportion of those who exhibited understanding was approximately two-fold higher than the proportion of those who exhibited understanding. A history of prior consultation with a dentist of different ethnic origin had no effect on the understanding. The dentists typically employ conventional communication in OHS.

In this study, we assessed the validity and reliability of the Indonesian version of the MUS questionnaire for measuring the appropriateness and effectiveness of communication between dentists and patients. Communication effectiveness is determined by understanding [3]. The MUS questionnaire provides numerical data and its reliability is indicated by interclass correlation coefficient. The MUS patient and dentist questionnaires showed either very good or excellent agreement. These results indicate that the MUS patient and dentist questionnaire has high reliability, so that it can be used repeatedly at the time of placement.

The result of the validity test of MUS dentist and MUS patients correlated with each Likert global rank and generally showed a good correlation. This result indicates a significant positive correlation between the MUS questionnaire and the global question of 5-points Likert test with respect to comprehension. The MUS patient and dentist questionnaire can measure the understanding of the information transacted in patient-dentist communications.

Measurement of understanding in OHS refers to measurement of the exchange of information about oral health. The exchange of information and views of health are influenced by culture $[5,7]$. The exchange of information during healthcare communication and doctor-patient interaction plays an important role in influencing mutual understanding [3]. Mutual understanding is the first step that should be followed by assessment of quality of communication and quality of service as perceived by patient's compliance or adherence [5].

The Indonesian version of MUS questionnaire can be used in multicultural clinical settings in both general health service and OHS to investigate the effect of cultural differences between the health service provider and patients on the level of mutual understanding achieved during the consultation. In addition, MUS can also be used to investigate the quality elements of medical consultation in everyday practice [3].

The nature of the assessment may have introduced an element of bias in the responses to the questionnaire. Ratings can be influenced by an individual's mood, past expe- 
riences, tendency to judge with more or less understanding level, influence of mass media, and the so-called "Hawthorne effect". These elements can be minimized by methodology, including sampling strategy, response rate, questionnaire formats, and data collection procedures, as another key factor is the quality of assessment instruments in terms of validity and reliability. A full study of these factors, especially regarding the validity of the questionnaire, should be done periodically. Further research should compare the shared understanding in dentists and patients belonging to different tribes and those belonging to the same tribe.

This study provides strong evidence in support of the reliability and validity of the Indonesian version of the MUS dentist and patient questionnaires for measurement of dentists' understanding, patients' understanding, and mutual understanding between dentists and patients during exchange of information and views in health or dental consultations in multicultural clinical settings.

This study limitation is Papua patients and dentists from Java only. Next study will be re-cross adaptation and re-test psychometry test of MUS in another ethnic group.

Thus, by further understanding the role of identity negotiation more in depth in the context of intercultural communication competence, individuals can learn to monitor the communication process and outcome more mindfully and, hopefully, with identity attunement. Not only effective interaction, but transactional and appropiate communication is important to achive mutual understanding [12].

\section{CONCLUSIONS}

This study provides strong evidence of the reliability and validity of the Indonesian MUS patient and dentist questionnaires in oral health services to measure mutual understanding.

\section{ACKNOWLEDGMENT}

This work was supported by Universitas Indonesia, Jakarta.

\section{CONFLICT OF INTEREST}

The authors declare no potential conflicts of interest with respect to the research, authorship, and/or publication of this article.

\section{References}

1. Paternotte E, van Dulmen S, Bank L, et al. Intercultural communication through the eyes of patients: experiences and preferences. Int J Med Educ 2017; 8: 170-175.

2. Schouten BC, Meeuwesen L, Harmsen HAM. GPs' interactional styles in consultations with Dutch and ethnic minority patients. J Immigr Minor Health 2009; 11: 468-475.
3. Harmsen JA, Bernsen RM, Meeuwesen L, et al. Assessment of mutual understanding of physician patient encounters: development and validation of a Mutual Understanding Scale (MUS) in a multicultural general practice setting. Patient Educ Couns 2005; 59: 171-181.

4. Harmsen H, Bernsen R, Meeuwesen L, et al. The effect of educational intervention on intercultural communication: results of a randomised controlled trial. Br J Gen Pract 2005; 55: 343-350.

5. Takayama T, Yamazaki Y, Katsumata N. Relationship between outpatients' perceptions of physicians' communication styles and patients' anxiety levels in a Japanese oncology setting. Soc Sci Med 2001; 53: 1335-1350.

6. Ha JF, Longnecker N. Doctor-patient communication: a review. Ochsner J 2010; 10: 38-43.

7. Kleinman A. Patients and healers in the context of culture: an exploration of the borderland between anthropology, medicine, and psychiatry. University of California Press, Berkeley 1980.

8. Paternotte E, Scheele F, van Rossum TR, et al. How do medical specialists value their own intercultural communication behaviour? A reflective practice study. BMC Med Educ 2016; 16: 222.

9. Paternotte E, Van Dulmen S, Van der Lee N, Scherpbier AJJA, Scheele F. Factors influencing intercultural doctor-patient communication: a realist review. Patient Educ Couns 2015; 98: 420-445.

10. Ivanoff CS, Yaneva K, Luan D, et al. A global probe into dental student perceptions about philanthropy, global dentistry and international student exchanges. Int Dent J 2017; 67: 107-116.

11. Delbanco T. Moving beyond Black and White. Ann Intern Med 2003; 139: 952.

12. Ting-Toomey S. Identity negotiation theory. In: Bennett J (ed.). Sage Encyclopedia of Intercultural Competence. Volume 1. Sage, Los Angeles 2015; pp. 418-422. 